\title{
Molecular cloning, expression and oxidative stress response of the vitellogenin Gene (AccVg) from Apis cerana cerana
}

\author{
Weixing ZHANG ${ }^{1}$, Zhenguo $\mathrm{LIU}^{1}$, Ming $\mathrm{ZHU}^{2}$, Lanting MA ${ }^{1}$, Ying WANG ${ }^{1}$, \\ Hongfang WANG ${ }^{1}$, Xingqi GuO ${ }^{2}$, Baohua $\mathrm{Xu}^{1}$ \\ ${ }^{1}$ College of Animal Science and Technology, Shandong Agricultural University, Tai' an, Shandong, People's Republic of \\ China \\ ${ }^{2}$ College of Life Sciences, Shandong Agricultural University, Tai'an, Shandong, People's Republic of China
}

Received 19 September 2016 - Revised 4 February 2017 - Accepted 24 February 2017

\begin{abstract}
Vitellogenin (Vg) is a yolk precursor protein in most oviparous females. However, Vg has not been studied in the Apis cerana cerana. In this work, the Vg gene of the A. cerana cerana has been cloned and sequenced. The gene codes for a protein consisting of 1770 amino acids in seven exons with a predicted molecular mass and isoelectric point of $200 \mathrm{kDa}$ and 6.46, respectively. Additionally, an 807-bp 5'-flanking region was isolated, and potential transcription factor binding sites associated with development and stress response were identified. Quantitative real-time PCR analysis showed that $\mathrm{AccVg}$ is highly expressed in pupae during different developmental stages. In addition, the expression of $\mathrm{AccVg}$ could be induced by cold $\left(4\right.$ and $\left.16{ }^{\circ} \mathrm{C}\right), \mathrm{CdCl}_{2}$, and pesticide treatment. Real-time quantitative PCR (RT-PCR) and western blot analyses indicated that AccVg transcription was induced by various abiotic stresses. Western blot was used to measure the expression levels of AccVg protein. Taken together, these results suggest that $\mathrm{AccVg}$ most likely plays essential roles in antioxidant defence and that it may be of critical importance to honey bee survival.
\end{abstract}

\section{Vitellogenin / Apis cerana cerana / RNA interference / Oxidative stress response / RT-PCR}

\section{INTRODUCTION}

In China, Apis cerana cerana is commonly found and plays an important role in agricultural economics and in the balance of regional ecologies as a pollinator of flowering plants. In honeybees, worker-worker division of labour is based on a process of behavioural maturation; for the first 2-3 weeks of adult life, workers perform tasks inside the hive, such as brood care and food storage, and as they become older, they progress to tasks outside, including foraging for pollen and nectar (Winston, 1987).

Electronic supplementary material The online version of this article (doi:10.1007/s13592-017-0503-9) contains supplementary material, which is available to authorized users.

Corresponding author: B. Xu, bhxu@sdau.edu.cn Handling Editor: Klaus Hartfelder
Organisms are exposed to a series of unfavourable environmental stressors on a nearly constant basis, including temperature change, heavy metals, pesticides, and ultraviolet (UV) radiation, all of which are believed to induce the formation of reactive oxygen species (ROS) (Lushchak, 2011; Kottuparambil et al., 2012). The generation of increased ROS and the production of oxidative damage are associated with induced thermal stress (Yang et al., 2010). ROS can be generated by all oxygenic organisms during aerobic metabolism (Dröge, 2002; Balaban et al., 2005). Therefore, understanding the antioxidant system of the honeybee and its ROS defence mechanisms has become a crucial issue for this industry.

Large lipid transfer proteins (LLTP) ${ }^{2}$ have multiple roles in animals, such as lipid transporters, inflammation suppressors, immunomodulators and blood coagulators. Vg is one of the 
ancient forms of these proteins, with an estimated 700 million-year history (Hayward et al., 2010).Vgs are mostly produced in the liver (vertebrates), hepatopancreas (crustaceans) or fat body (invertebrates); secreted into the blood and taken up by targets via receptor-mediated endocytosis (Spieth et al., 1991; Tufail and Takeda, 2008; Tseng et al., 2001). Honeybee $\mathrm{Vg}$, known as a conserved yolk precursor protein, is a $180-\mathrm{kDa}$ glycolipoprotein synthesized in fat body cells and released to the haemolymph (the insect blood) (Wheeler and Kawooya, 1990; Fleig, 1995). In egg-laying queens, $\mathrm{Vg}$ constitutes over $50 \%$ of total haemolymph proteins and is taken up by developing oocytes (Hartfelder and Engels, 1998). In the haemolymph of nurse bees, Vg comprise 30$50 \%$ of total proteins and impacts both antioxidant and immune function (Amdam et al., 2004; Seehuus et al., 2006; Corona et al., 2007). Additionally, $\mathrm{Vg}$ is present in the haemolymph of young worker bees, as well as in drones where it may account for up to $5 \%$ of total haemolymph proteins (Trenczek et al., 1989). Vg has an antioxidant function in workers (Seehuus et al., 2006). Knockdown of Vg expression in workers causes decreased resistance to oxidative stress. The Vg titre positively correlates with the honeybee's oxidative stress tolerance because the antioxidant property of $\mathrm{Vg}$ shields other haemolymph molecules from reactive oxygen species (ROS) (Seehuus et al., 2006). Additionally, a causal link between honeybee vitellogenin activity and lifespan is supported by the zincbinding capacity of the protein product (Amdam et al., 2004), which also suggests an antioxidant function (Amdam and Omholt, 2002; Goto et al., 1999; Quinlan et al., 2005).

The vitellogenins have been studied extensively in a wide variety of animals, both vertebrates and invertebrates, including insects. In the present study, we isolated and characterised the $\mathrm{Vg}$ in A. cerana cerana (AccVg). Additionally, we analysed the expression of $\mathrm{Vg}$ during different developmental stages and under various abiotic stress conditions. Finally, we investigated the activity of $\mathrm{AccVg}$ under various stress conditions, including cold, insecticides and heavy metal exposure.

\section{MATERIALS AND METHODS}

\subsection{Biological specimens and treatments}

The Chinese honeybees, A. cerana cerana, maintained at the experimental apiary of Shandong Agricultural University (Tai'an, Shandong Province, China), were used in the experiments following different treatments. Newly emerged workers within an 8-h period were tagged with numbered Opalith plates (Graze, Germany) at emergence from hives. When the tagged workers were 15 days old, these were called foragers. They were captured and randomly divided into 6 groups, each group with 3 independent biological samples of 150 individuals, caged in wooden cage during the treatment which were kept in an incubator at a constant temperature $\left(31^{\circ} \mathrm{C}\right)$ and humidity $(60 \%)$ and fed with pollen-and-sucrose solutions (Pham-Delègue et al., 2000).

For the environmental stress analysis, the worker bees were treated with cold $\left(4^{\circ} \mathrm{C}\right)$ or heat $\left(42{ }^{\circ} \mathrm{C}\right)$ for the indicated times. For the pesticide treatments, cyfluthrin, phoxim and paraquat were diluted to a final concentration of $20 \mathrm{mg} / \mathrm{L}$. The diluted liquid $(200 \mu \mathrm{L})$ was sprayed into gauze and the gauze was put in the wooden cage. For the heavy metal treatment, worker bees were fed with pollen-and-sucrose solutions containing $\mathrm{CdCl} 2$ $(1.0 \mu \mathrm{g} / \mathrm{mL})$. The control group was fed only the pollen-and-sucrose solution. The bees were kept in the cages and exposed to the different treatments for up to $4 \mathrm{~h}$. For different developmental stages and tissues, untreated larvae and adult workers were harvested for gene expression analysis. A total of 60 15-day-old workers were anaesthetised on ice, and the brains, thoraces and guts were dissected. Three individual bees were dissected for each stage. All of the bees were flash-frozen in liquid nitrogen at an appropriate time and stored at $-80{ }^{\circ} \mathrm{C}$ for RNA or DNA extraction.

\subsection{RNA extraction, cDNA synthesis and genomic DNA isolation}

Total RNA was extracted using the RNAiso Plus (TaKaRa, Dalian, China) according to the manufacturer's instructions, followed by RNase- 
free DNase I treatment. Single-stranded cDNA was synthesized using Transcript ${ }^{\circledR}$ All-in-One First-Strand cDNA Synthesis SuperMix for qPCR (TransGen Biotech, Beijing, China) at $42{ }^{\circ} \mathrm{C}$ for $15 \mathrm{~min}$, followed by $85^{\circ} \mathrm{C}$ for $15 \mathrm{~s}$. Genomic DNA was isolated from newly emerged workers using an EasyPure Genomic DNA Extraction Kit according to the manufacturer's instructions (TransGen Biotech).

\subsection{Primers}

The primers used in the present study were listed in Table I.

\subsection{Isolation of the full-length cDNA and the genomic sequence of $A c c V G$}

To obtain the fragment of AccVg cDNA, special primers (VG1/VG2, VG3/VG4, VG5/VG6) were designed and synthesized (Shanghai Sangon Biotechnological Company, Shanghai, China) by the conserved regions of the $\mathrm{Vg}$ from A. mellifera, Nasonia vitripennis and Locusta migratoria. Supplementary Fig. 1 shows the position of primers in Vg genes. The cDNA of newly emerged workers was used for cloning the fulllength cDNA. On the basis of the sequence of the cloned conservative fragments, specific primers $(3 \mathrm{~N} 1,3 \mathrm{~N} 2,5 \mathrm{~N} 1,5 \mathrm{~N} 2)$ were designed and synthesized for 3'- and 5'- rapid amplification of cDNA ends (RACE). All the PCR products were purified using a gel extraction kit (Solarbio, Beijing, China). The PCR products were ligated into pEasyT3 vector (TransGen Biotech) and transformed into Escherichia coli strain DH5 $\alpha$ for sequencing.

To isolate the AccVg gene, three pairs of specific primers $(\mathrm{N} 1 / \mathrm{N} 2, \mathrm{~N} 3 / \mathrm{N} 4$ and $\mathrm{N} 5 / \mathrm{N} 6)$ were designed based on the AccVg cDNA sequence. The PCR reaction uses $1 \mu \mathrm{L}$ genomic DNA as template, $0.25 \mu \mathrm{L}$ Tap DNA polymerase, $2.5 \mu \mathrm{L}$ PCR buffer, $1 \mu \mathrm{L}$ dNTP, $1 \mu \mathrm{L}$ forward primer and reverse primer and $18.25 \mu \mathrm{L} \mathrm{ddH}_{2} \mathrm{O}$ in a $25 \mu \mathrm{L}$ volume with the following cycling conditions: initial denaturation program $\left(94{ }^{\circ} \mathrm{C}\right.$ for $10 \mathrm{~min}$ ), followed by 35 cycles of $94{ }^{\circ} \mathrm{C}$ for $40 \mathrm{~s}, 55^{\circ} \mathrm{C}$ for $40 \mathrm{~s}$ and $72{ }^{\circ} \mathrm{C}$ for $120 \mathrm{~s}$. Finally, the PCR products were separated, purified, ligated into pEasy-
T3 vector and transformed into E.coli DH5 $\alpha$ for sequencing.

\subsection{Amplification of the $5^{\prime}$-flanking region of $\mathrm{AccVg}$}

The 5'-flanking region of $A c c V g$ was obtained using the inverse polymerase chain reaction (IPCR). The genomic DNA was digested with the restriction endonuclease $N s i$ I at $37^{\circ} \mathrm{C}$ overnight, self-ligated to form circles using T4 DNA ligase (TaKaRa, Dalian, China) and used as a template for IPCR. Two pairs of primers (IF1/ IR1, IF2/IR2) were designed and synthesised based on the genomic DNA sequence. Finally, the sequence of the 5'-flanking region of $\mathrm{AccVg}$ was used to design specific primers (P1/P2) for direct PCR amplification from genomic DNA. The PCR reaction uses $1 \mu \mathrm{L}$ genomic DNA as template, $1 \mu \mathrm{L}$ dNTP, $1 \mu \mathrm{L}$ forward primer and reverse primer, $0.25 \mu \mathrm{L}$ Tap DNA polymerase, $2.5 \mu \mathrm{L}$ PCR buffer and $18.25 \mu \mathrm{L} \mathrm{ddH}_{2} \mathrm{O}$ in a $25 \mu \mathrm{L}$ volume with the following cycling conditions: initial denaturation program $\left(94^{\circ} \mathrm{C}\right.$ for $10 \mathrm{~min}$ ), followed by 35 cycles of $94{ }^{\circ} \mathrm{C}$ for $40 \mathrm{~s}, 50{ }^{\circ} \mathrm{C}$ for $40 \mathrm{~s}$ and $72{ }^{\circ} \mathrm{C}$ for $90 \mathrm{~s}$. All of the PCR products were purified, ligated into the pMD19-T vector and sequenced.

\subsection{Bioinformatic and phylogenetic analyses}

The theoretical isoelectric point and molecular weight of AccVg were predicted using ExPASy online software (http://web.expasy.org/compute pi/). The sequences of $\mathrm{AccVg}$ homologous with other species were retrieved using the NCBI server (http://blast.ncbi.nlm.nih.gov/Blast.cgi) and aligned using DNAMAN version 5.2.2 (Lynnon Biosoft, Quebec, Canada). The phylogenetic analysis was carried out using the Molecular Evolutionary Genetics Analysis (MEGA version 4.1) (http://www.megasoftware.net/) based upon the neighbour-joining method. The SWISS-MODEL (http://swissmodel.expasy.org/) online software was used to predict the tertiary structure of AccVg. The TFSEARCH website (http://www. cbrc.jp/research/db/TFSEARCH.html) was used to predict the transcription factor binding sites. 
Table I. PCR primers in this study

\begin{tabular}{|c|c|c|}
\hline Abbreviation & Primer sequence $\left(5^{\prime} \rightarrow 3^{\prime}\right)$ & Description \\
\hline VG1 & ATGTTGCTACTTCTAACGCTT & cDNA sequence primer, forward \\
\hline VG2 & CGTAGAGGCTGTTGTCGTGT & cDNA sequence primer, reverse \\
\hline VG3 & CAACACCGCCGCCACCTTAT & cDNA sequence primer, forward \\
\hline VG4 & TTGCTGTTCGCCTGCTTGTCCG & cDNA sequence primer, reverse \\
\hline VG5 & GAGGAAATGTGGGAACTGAT & cDNA sequence primer, forward \\
\hline VG6 & ACGAAAGAAAGCGACAAAGT & cDNA sequence primer, reverse \\
\hline $5 \mathrm{P} 1$ & CGGGCATCATACAAACCACAT & 5'-RACE reverse primer, outer \\
\hline $5 \mathrm{P} 2$ & TGGTGGTCGGTGAACAAACT & 5'-RACE reverse primer, inner \\
\hline AAP & GGCCACGCGTCGACTAGTAC(G)14 & Abridged Anchor Primer \\
\hline AUAP & GGCCACGCGTCGACTAGTAC & Abridged universal amplification primer \\
\hline $3 \mathrm{P} 1$ & AAGGAGACCGACGACAAGA & 3'-RACE forward primer, outer \\
\hline $3 \mathrm{P} 2$ & AAGAACGAGGCCGCGATGAA & 3'-RACE forward primer, inner \\
\hline B26 & GACTCTAGACGACATCGA(T) 18 & 3'-RACE universal primer, outer \\
\hline B25 & GACTCTAGACGACATCGA & 3'-RACE universal primer, inner \\
\hline N1 & ATGTTGCTACTTCTAACGCTT & Genomic sequence primer, forward \\
\hline $\mathrm{N} 2$ & CGTAGAGGCTGTTGTCGTGT & Genomic sequence primer, reverse \\
\hline N3 & CAACACCGCCGCCACCTTAT & Genomic sequence primer, forward \\
\hline N4 & TTGCTGTTCGCCTGCTTGTCCG & Genomic sequence primer, reverse \\
\hline N5 & GAGGAAATGTGGGAACTGAT & Genomic sequence primer, forward \\
\hline N6 & ACGAAAGAAAGCGACAAAGT & Genomic sequence primer, reverse \\
\hline IF1 & GGTCTGAGCAGTTGCACGCTG & Inverse PCR forward primer, outer \\
\hline IF2 & TCTAAACGAAACAAAGCGGCA & Inverse PCR reverse primer, outer \\
\hline IR1 & TCGCAATCTTCATCGTTGTA & Inverse PCR forward primer, inner \\
\hline IR2 & GTTCAAGTCCTCGGTGATAG & Inverse PCR reverse primer, inner \\
\hline P1 & AAAGCGGGGTACAGGATG & Promoter special primer, forward \\
\hline P2 & GGTGAGCAACGCCTTTA & Promoter special primer, reverse \\
\hline VG-1 & 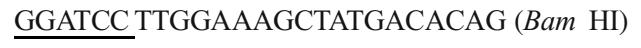 & Protein expression primer, forward \\
\hline VG-2 & CTCGAGCTGGCTCTGGCTAACTTTACC (Xho I) & Protein expression primer, reverse \\
\hline RT1 & TCGGTGACTACGAGCAGGAT & Real-time PCR primer, forward \\
\hline RT2 & GGGCAGAGGTTTGGACACT & Real-time PCR primer, reverse \\
\hline$\beta-s$ & CCGTGATTTGACTGACTACCT & Standard control primer, forward \\
\hline$\beta-x$ & AGTTGCCATTTCCTGTTC & Standard control primer, reverse \\
\hline
\end{tabular}

\subsection{Production of recombinant AccVg and antibody preparation}

Using the primers VG-1/VG-2 (Table I), a 1128-bp cDNA fragment was obtained (a part of the whole cDNA, 920-1295 amino acids), cloned into the expression vector PET-28a $(+)$, and the recombinant plasmid pET-28a (+)-AccVg was transformed into E. coli BL21 (ED3), which was grown at $37^{\circ} \mathrm{C}$ in $10 \mathrm{~mL}$ of Luria-Bertani broth (LB) supplemented with $40 \mu \mathrm{g} / \mathrm{mL}$ kanamycin. When the cell density reached $0.6 \mathrm{OD}_{600}$, isopropyl-1-thio- $\beta$-galactopyranoside (IPTG) was added to a $0.5 \mathrm{mM}$ final concentration to induce the expression of the recombinant protein. Cells were centrifuged at $6500 \times g$ at $4{ }^{\circ} \mathrm{C}$ for $10 \mathrm{~min}$, after incubation at $37^{\circ} \mathrm{C}$ for $4 \mathrm{~h}$. The pellet was resuspended in $10 \mathrm{~mL}$ of lysis buffer, 
sonicated and centrifuged again at $4{ }^{\circ} \mathrm{C}$ at $13,000 \times g$ for $15 \mathrm{~min}$. The recombinant protein was purified using the HisTrap ${ }^{\text {TM }}$ FF column (GE Healthcare, Uppsala, Sweden) according to the manufacturer's instructions. Finally, the purified protein was separated by sodium dodecyl sulphate polyacrylamide gel electrophoresis (SDS-PAGE) on $7.5 \%$ acrylamide gels. The purified protein was more than $95 \%$ pure by SDS-PAGE gel analysis. The purified protein was injected subcutaneously into New Zealand White male rabbits weighing 2-3 $\mathrm{kg}$ for generation of antibodies (Bitondi and Simoes, 1996).

\subsection{Quantitative RT-PCR}

To determine the expression patterns of the AccVg in A. cerana cerana, quantitative RTPCR was used. Amplification of the actin transcript (Gene ID: LOC108003299) was used as a sample control (Guidugli et al., 2005). The special primers RT1/RT2 and the actin gene primers actin-f/ actin-r are listed in Table I. The quantitative RT-PCR was carried out using the TransStart ${ }^{\circledR}$ Tip Green qPCR SuperMix (TransGen Biotech) in a $20 \mu \mathrm{L}$ volume on a CFX96 ${ }^{\mathrm{TM}}$ Real-time System (Bio-Rad) with the following cycling conditions: denaturation program $\left(94{ }^{\circ} \mathrm{C}\right.$ for $30 \mathrm{~s}$ ), followed by 40 cycles of $94{ }^{\circ} \mathrm{C}$ for $5 \mathrm{~s}, 55^{\circ} \mathrm{C}$ for $15 \mathrm{~s}$ and $72{ }^{\circ} \mathrm{C}$ for $10 \mathrm{~s}$. At the end of the amplification phase, a melting curve analysis was performed with a minimum of $60^{\circ} \mathrm{C}$ to a maximum of $94^{\circ} \mathrm{C}$ and $0.2^{\circ} \mathrm{C}$ increases for every $15 \mathrm{~s}$ to test the specificity and identity of the qRT-PCR products. Negative controls without the addition of RT enzyme were run to check for contamination by genomic DNA. To check reproducibility, each SYBR green assay was performed in triplicate, and for each data point, we analysed three independent biological samples. The relative transcript quantities of the $\mathrm{Vg}$ and actin genes were calculated using the comparative $\mathrm{Ct}$ method (Applied Biosystems, User Bulletin 2; Livak and Schmittgen, 2001; Tang-Feldman et al., 2006). The statistical analysis of the differences was determined by Duncan's multiple range test using Statistical Analysis System (SAS) version 9.1 software.

\subsection{Western blot analysis}

Whole-body proteins were extracted using RIPA Lysis Buffer (Beyotime, Jingsu, China) according to the manufacturer's instructions and quantified with BCA Protein Assay Kit (Beyotime). Soluble proteins were separated by SDS-PAGE on $7.5 \%$ acrylamide gels, transferred to a polyvinylidene fluoride (PVDF) membrane (Millipore, Immobilon $0.22 \mu \mathrm{m}$ ) and processed for western blot. Membranes were treated with the anti-AccVg serum against $\mathrm{AccVg}$ protein raised in rabbits, used at a dilution of 1:500. The Goat Anti-Rabbit lgG, HRP conjugated (CWBIO, China), was used as the secondary antibody at a 1:2000 dilution. Proteins were visualized using chemiluminescent peroxidase substrate (Beyotime Biotechnology, Shanghai, China), and then the blots were quantified using a Fusion Fx system (UVP, America) and the analysis software FusionCapt Advance system (UVP, America).

\subsection{Statistical analysis}

All data are reported as the mean \pm standard error (SE), unless otherwise stated. The SAS version 9.1 was used for data analysis. Differences between the treatment groups were analysed using the Duncan's multiple range test and analysis of variance (ANOVA) to identify homogenous groups of treatments. $P$ values $<0.05$ were considered statistically significant. The different letters above bars denote significant differences between the treatment groups.

\section{RESULTS}

\subsection{Molecular characterization of AccVg and features of the recombinant $A c c V g$ protein}

The full-length AccVg cDNA sequence (GenBank accession number: kp398512) was $5411 \mathrm{bp}$, including $5313 \mathrm{bp}$ in the open reading frame, $26 \mathrm{bp}$ in the $5^{\prime}$-untranslated region and $72 \mathrm{bp}$ in the 3 '-untranslated region. The ORF encodes a polypeptide of 1770 amino acids with a calculated molecular mass of $200 \mathrm{kDa}$ and a theoretical isoelectric point (PI) of 6.46, 
respectively (Fig. S2). Multiple sequence alignment revealed high amino acid identity $(89.05 \%)$ between $\mathrm{AmVg}$ and $\mathrm{AccVg}$ (Fig. S3).

To investigate the evolutionary relationship between $A c c V g$ and other $V g$ s from different species, a phylogenetic tree of $V g$ s was constructed. Of the eight insect species for which $V g$ s were examined, AccVg was categorised into the Hymenoptera order. As shown in Fig. 1, phylogenetic analysis revealed that $\mathrm{AccVg}$ was more closely related to $\mathrm{AmVg}$ than to homologues in other species. The SWISS-MODEL was used to predict and reconstruct the three-dimensional structure (Fig. S4).

The molecular mass of $\mathrm{AccVg}$ protein is so large that preparation of recombinant plasmid PET-28a (+)-AccVg was difficult. We expressed a portion of the protein (920-1295 aa) to prepare antibodies against AccVg. SDS-PAGE analysis showed that the recombinant protein was soluble and had a molecular mass of approximately $50 \mathrm{kDa}$ (Fig. 2). The entire western blot of the specific antibody against $A c c V g$ is shown in Supplementary Fig. 5.

\subsection{Genomic structure and the characterization of the 5'-flanking region of $\mathrm{AccVg}$}

By cloning and assembling cDNA fragments and intron, we obtained the AccVg genomic sequence. To better understand the organization of the regulatory elements, primers (IF1/IF2 and IR1/IR2) were used to obtain the 5' flanking region of AccERR, using inverse PCR (IPCR). Briefly, total genomic DNA was digested with

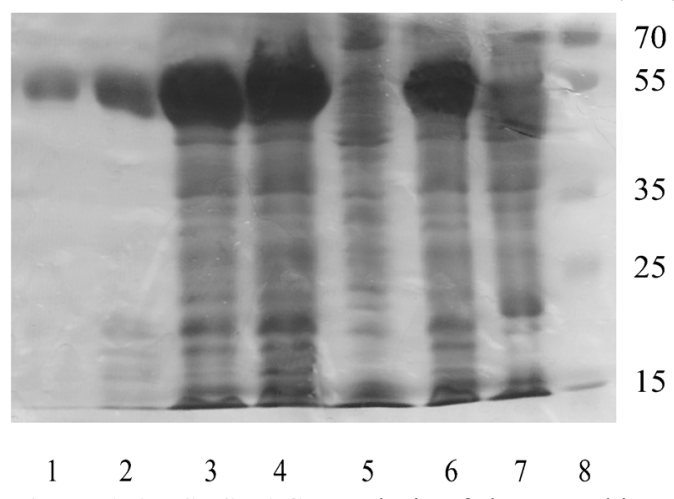

Figure 2 An SDS-PAGE analysis of the recombinant AccVg protein. Lane 8 protein marker. Lines 1 and 2 purified recombinant protein. Lines 3 and 4 suspension of recombinant part of $A c c V g$, respectively. Lines 6 and 7 induced and non-induced overexpression of pET-28a (+)-AccVg in BL21, respectively. Line 5 induced overexpression of pET-28a (+) in BL21.

the restriction endonuclease $\mathrm{Nsi}$ I at $37{ }^{\circ} \mathrm{C}$ overnight, self-ligated to form circles using T4 DNA ligase (TaKaRa, Dalian, China) and used as a template for IPCR. The genomic DNA sequence of Acc Vg contains 7064 bp, which includes seven exons that are separated by six introns with high AT content and flanked by the $5^{\prime}$ splice donor GT and the $3^{\prime}$ splice acceptor AG signals. The introns have typical eukaryotic characteristics with high AT content.

To further analyse the relationship of $\mathrm{AccVg}$ with other $V g \mathrm{~s}$, the genomic structures of E. formosa $\mathrm{Vg}$, A. mellifera $\mathrm{Vg}, \mathrm{T}$. amboinensis $V g$ and $A$. aegypti $V g$ were compared, as shown

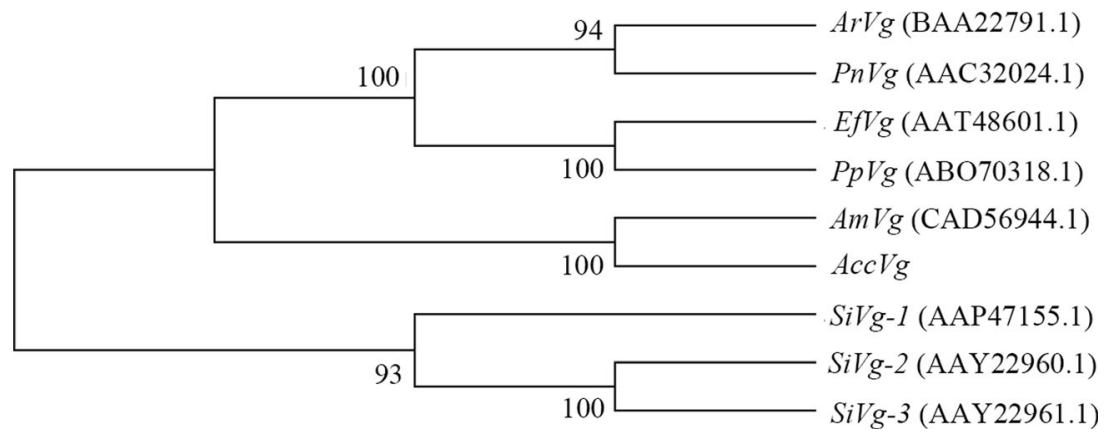

Figure 1 Phylogenetic relationships on the basis of the amino acid sequences between $V g$ s from different insect species. 
in Fig. 3. The analysis showed that $E f V g, A m V g$ and $A c c V g$ contain seven exons and possess the longest stretch of sequence similarity, whereas $\mathrm{TaVg}$ and $\mathrm{AaVg}$ contained only three exons. The numbers, sizes and relative positions of all the introns of $V g \mathrm{~s}$ in different species are different.

To better understand the molecular mechanism of the transcriptional regulation of $\mathrm{AccVg}$, an 833bp 5'-flanking fragment was isolated. A series of putative $c i s$-acting elements was predicted using TFSEARCH software. A series of putative cis acting elements was also predicted after analysing the promoter sequence of $\mathrm{AccVg}$. Some heat shock elements, which are the binding sites for heat shock factor (HSF), were also found (Ding et al., 2005). Several important cis-acting elements involved in embryo or organ development, including broad-complex (BR-C), NIT 2 and Cdx A were identified (Zhang et al., 2013). Additionally, the Ovo homologue-like transcription factor (TF) binding site involved in epidermis differentiation was predicted. The CAAT-box, which is critical for transcription, was found in the 5'flanking region of the promoter. Other putative transcription factors are shown in Fig. 4. These putative transcription factor binding sites imply that AccVg may be subject to developmental regulation.

\subsection{Developmental and tissue expression profiles of $A c c V g$}

$V g$ expression has been discovered not only in adults but also in younger stages and late pupae in A. mellifera (Engels et al., 1990; Hartfelder and
Engels, 1998; Barchuk et al., 2002; Piulachs et al., 2003; Guidugli et al., 2005). Thus, we were interested in studying whether $V g$ is expressed in the developmental stages (larvae, pupae, adults) and in various tissues of $A$. cerana cerana, using total RNA for qPCR analysis and protein isolates in western blots. In workers, AccVg mRNA expression slowly rose until reaching a maximum at the third larval instar, decreased in pre-pupae, became practically undetectable in pupae and increased again in newly emerged workers (Fig. 5a) $(\mathrm{F}=99.16 ; P<0.05)$. The expression of $\mathrm{AccVg}$ in different tissues is shown in Fig. 5b. In terms of various tissues, the highest amount of $\mathrm{AccVg}$ was detected in the abdominal epidermis; because, the epidermis contains more fat body cells that are known to produce abundant vitellogenin, more than do other tissues. The lowest quantity transcript was detected in the brain $(\mathrm{F}=101.13$; $P<0.05)$.

\subsection{Expression patterns of $\mathrm{AccVg}$ under different environmental stresses}

Previous studies have indicated that the expression of $V g$ could be induced by abiotic stress. To better understand whether $A c c V g$ is involved in various abiotic stress responses, the relative expression of $A c c V g$ under cold $\left(4^{\circ} \mathrm{C}\right)$, heat $\left(42^{\circ} \mathrm{C}\right)$, heavy metal and insecticide (cyfluthrin, phoxim, paraquat) exposure was determined by qPCR. Following the cold $\left(4^{\circ} \mathrm{C}\right)$ treatment, the $\mathrm{AccVg}$ transcript level was slightly elevated (Fig. 6a) $(\mathrm{F}=6.10 ; P<0.05)$, but a significant alteration of $A c c V g$ expression was observed after the heat $\left(42{ }^{\circ} \mathrm{C}\right)$ treatment (Fig. $\left.6 \mathrm{~b}\right)(\mathrm{F}=155.88$;

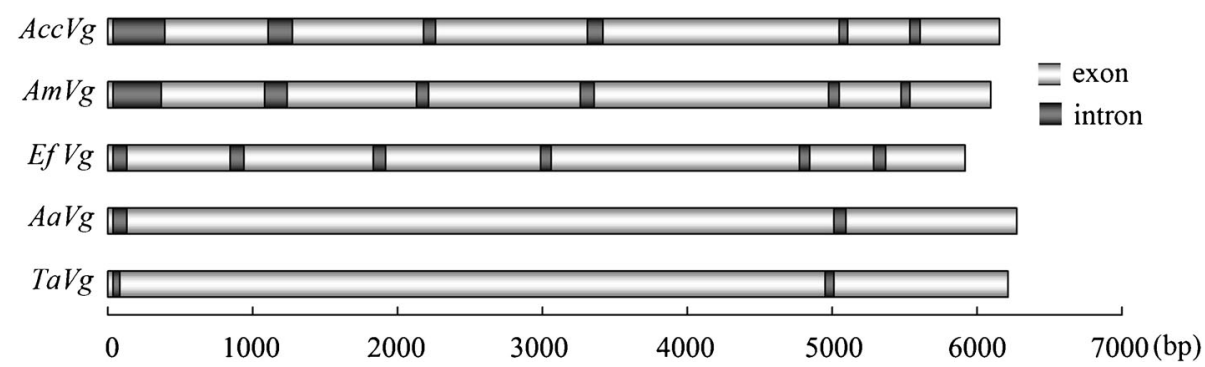

Figure 3 The DNA structures of Vgs. The length extrons and introns of genomic DNA of AccVg, AmVg (NC_007073), EfVg (AY553878), AaVg (AY691327) and TaVg (AY691326) are shown according to the scale below. 
AAAGCGGGGTACAGGATGTAAATATCTAGAATTCAAAGTTTAACATTGAAAATATCATTGCCGTGAACT Ovo homolog-like transcription factor Ovo homolog-like transcription factor

CATGACACTCGTGTTATTTTATATATCATATTTTTTAATCAATCGTAATATTTACGAATGAAAATCTCG CAAT box

Ovo homolog-like transcription factor

$\mathrm{Cdx} \mathrm{A}$

IGTTTACTTGCATTTATATTAACGATAA ATTTTTTTTATA TCATATTCA TCA TATTGTA TCATTATCAC NIT 2 CAAT box

ATTACGAATATTTCGATGATTTTGTATCAAGATACT'GATCAATGTATCAGAGAGATTTTTTTTTTT'TTA ATTATTAAAAGTAATCATAAAATTAAAATAAAATCTTAATTGTATCTTAATCATAGTAATTATTAAAAA TTATAAAATATTTTAAATTAAAATCTAGGGGTGAAAATTCAAAGTGGTAAGCAAAGATTAACTTTAAGA TTAAAAATGCAACTTTTAAACTATTT'GCTAAAAA TAAATT TTTCACTTT TT'TAT'GTAAAAT'TATGTTAT CAAT box HSF

GTAATTTTATACTTCTCGATTATATATCGACCTTGTAATCGCATCTTAAAAATCAATTTAAAGAAAACA CAAT box Cdx A

GATATTATTA TCACGCATTCGAAGACAATAATA TTGAATCATTTATAGTTACATGTTTTTTTCAAAAGA HSF

TATTATTATTAGCTGTTGTTACGCCAAACACAGATTATAGACATGCTAGAAACGAGCGGAGAGATAAAA -118 TAAAAAAGATAAAATAAAAAAA BATAGACATAATAACGTGTTTCAATCTAGCAAAAGAGAAAAATGCTG $+1$

ATTTAGCATAAATAAGATATAAATTGCTAAGCCGAATCAAATGCATCGT'TACTT TCTCGAAAGTTGTCT $\longrightarrow$ Transcription start

TCAACATGTTGCT

$\longrightarrow$ Translation start

Figure 4 The characterization of 5'-flanking region in AccVg. Predicted transcription factor binding sites are boxed . The transcription start site and translation start site are marked with an arrowhead.

$P<0.05)$. As shown in Fig. 6c, expression of AccVg was enhanced by heavy metal $\left(\mathrm{CdCl}_{2}\right)$ treatment and reached the highest transcript level at $9 \mathrm{~h}$ of exposure $(\mathrm{F}=42.39 ; P<0.05)$. With regard to the insecticide treatments, $A c c V g$ was induced by cyfluthrin, phoxim, and paraquat and reached the highest transcript level at 4, 2 and $2 \mathrm{~h}$, respectively. The results shown are relative to control levels at time 0 ; nonetheless, we also checked the levels of AccVg expression in control bees during the duration of the treatment period to make sure that there was no change other than the one induced by the treatments. These results suggested that $\mathrm{AccVg}$ may be involved in abiotic stress response.

\subsection{Western blot analysis}

To gain a comprehensive understanding of the molecular mechanisms underlying the effects of abiotic stress, the protein levels of $\mathrm{AccVg}$ were examined by Western blot analysis. An antiAccVg antibody was generated and used to detect AccVg. As shown in Fig. 7, when workers were exposed to heat $\left(42{ }^{\circ} \mathrm{C}\right), \mathrm{CdCl}_{2}$, phoxim or paraquat, the expression of $\mathrm{AccVg}$ was significantly increased. In terms of mRNA, the high mRNA levels seen in the figure correspond to the low Vg amounts detected in the western blots, which suggests the existence of mechanisms operating on the translatability and/or stability of Vg mRNA.

\section{DISCUSSION}

Previous studies demonstrated that Vg plays an important role in energy metabolism and contributes to growth and development in invertebrates. A growing number of studies have been conducted on the roles of $\mathrm{Vg}$ in response to adverse stress. However, no studies had systematically imitated the variety of stresses that animals may experience during their life. In this study, the honeybees were foragers obtained from the comb, which were always subjected to the outside environment. Several stresses that honeybees may encounter during their lifespan were imitated, and the resulting changes were explored.

The phylogenetic tree constructed in this study indicated that the $\mathrm{Vg}$ amino acid sequence has been conserved throughout evolution and that $A c c V g$ is in the typical $V g$ cluster, sharing the highest degree of homology with $\mathrm{Vg}$ of 

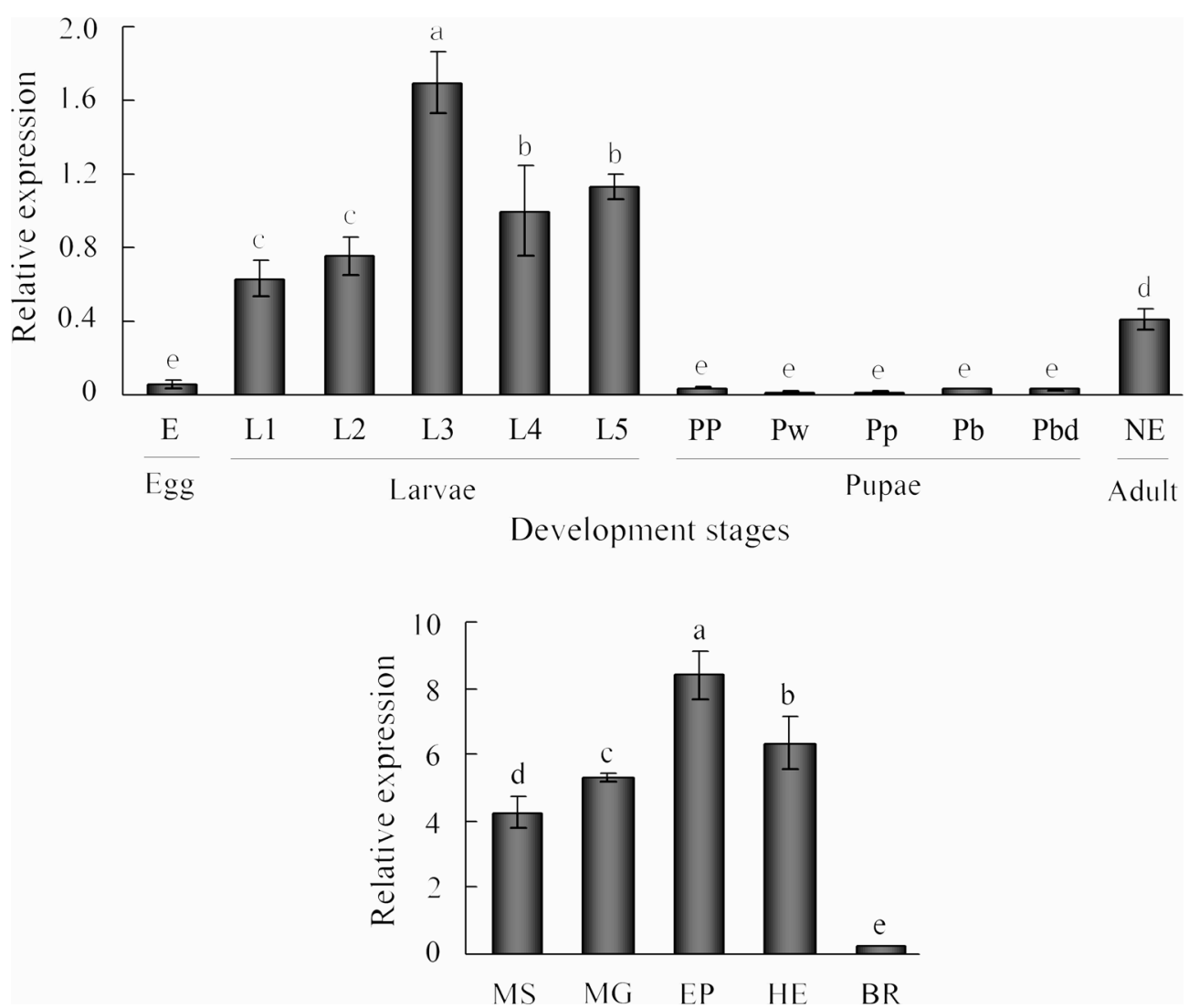

Figure $5 \mathrm{Vg}$ expression during different developmental stages and in tissues of $A$. cerana cerana workers. a Quantitative RT-PCR analysis of $V g$. L1, L2, L3, L4, L5 = larval stage; $P P, \mathrm{Pw}, \mathrm{Pp}, \mathrm{Pb}, \mathrm{Pbd}=$ pupal phase ( $P P$ prepupae, $P w$ white-eyed pupae, unpigmented cuticle, $P p$ pink-eyed pupae, unpigmented cuticle, $P b$ brown-eyed pupae, unpigmented cuticle, $P b d$ brown-eyed pupae, dark pigmented cuticle), and adults ( $N E$ newly emerged workers) according to Michelette and Soares (1993). b AccVg expression profiles in the brain (BR), epidermis $(E P)$, muscle $(M S)$, haemolymph $(H P)$, midgut $(M G)$.

A. mellifera. Taken together, these results indicate that the gene we isolated is a bona fide $V g$ homologue.

The timing and level of gene expression are determined by the promoter region, both of which depended on the role the gene plays. To predict the putative roles of $\mathrm{AccVg}$, an 807-bp 5'-flanking region was cloned and putative binding sites for transcription factors were predicted. The region contained a large number of putative binding sites for transcription factors involved in development and the response to environmental stress, such as a BR-C binding site and an Ovo homologue-like TF binding site, which are involved in the early stages of tissue development and growth. This result prompted us to study the putative roles of $\mathrm{AccVg}$. To achieve this goal, we analysed the transcript levels of the gene encoding AccVg during all development stages, in different tissues and under various abiotic stresses. Stage-specific expression analysis indicated that the $A c c V g$ gene is expressed throughout the lifecycle of bees and that the highest accumulation of AccVg was detected in the third larval instar (Fig. 5a). AccVg expression was still very high in adults, although lower than in the third larval instar. At the egg and pupal stages, $\mathrm{AccVg}$ expression was the lowest. This finding suggests that during the larval stages, the function of $A c c V g$ is to control the rate of development, whereas during the pupal stages, 
A

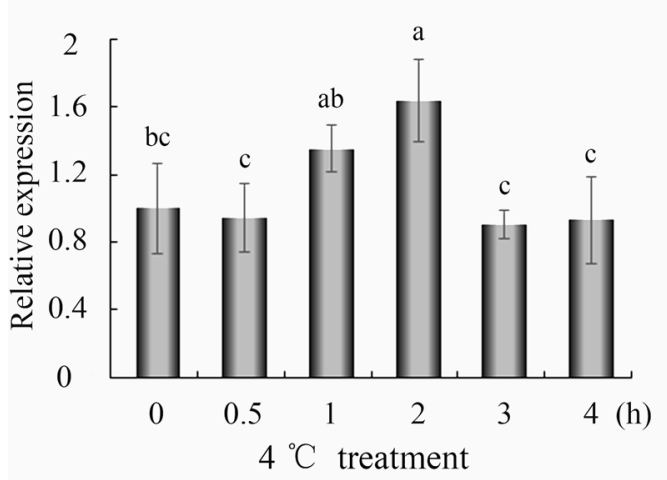

C

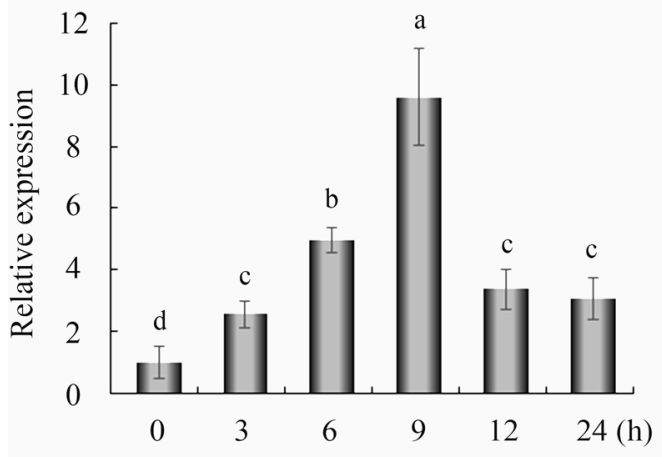

$\mathrm{Cdcl}_{2}$ treatment

E

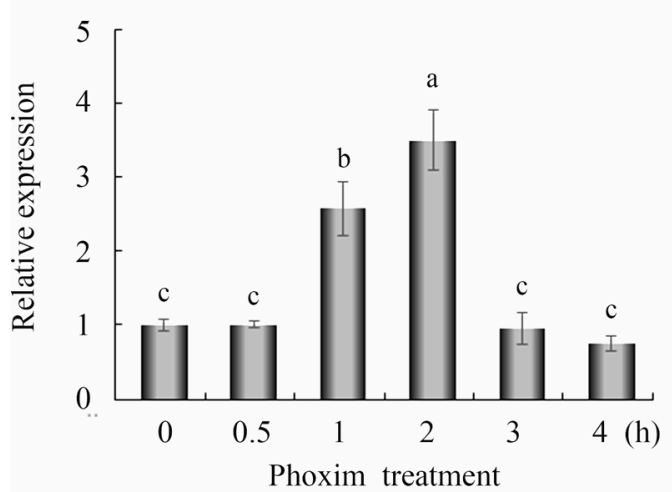

B

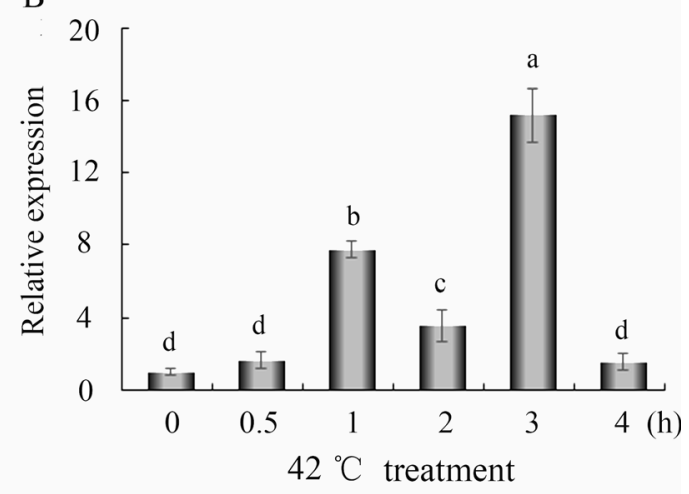

D

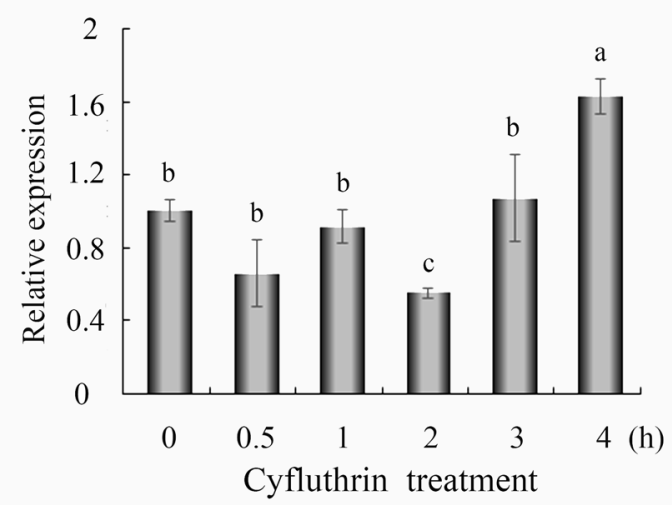

F

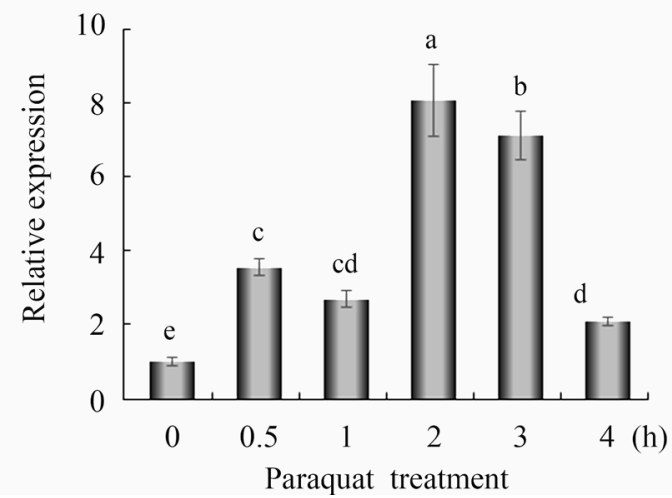

Figure $6 \mathrm{AccVg}$ transcript levels under different stress condition. These conditions include cold $\left(4{ }^{\circ} \mathrm{C}\right)(\mathbf{a})$, heat $\left(42{ }^{\circ} \mathrm{C}\right)(\mathbf{b}), \mathrm{Cdcl}_{2}(\mathbf{c})$, cyfluthrin $(\mathbf{d})$, phoxim (e) and paraquat (f). The RT-qPCR results for the treatments were normalized to bees without any stress treatment $(0 \mathrm{~h})$ for $\mathbf{a}-\mathbf{f}$. The different letters above the columns indicate significant differences $(P<0.05)$ according to Duncan's multiple range tests.

the expression of $A c c V g$ decreased. The stagespecific expression of $A c c V g$ indicated that $\mathrm{AccVg}$ might play roles when the bees were exposed to adverse stress.
Additionally, the mRNA level of AccVg was also characterized after various abiotic stress treatments (Fig. 6). When the honeybees were treated with $\mathrm{CdCl}_{2}, A c c V g$ expression was slightly 
A

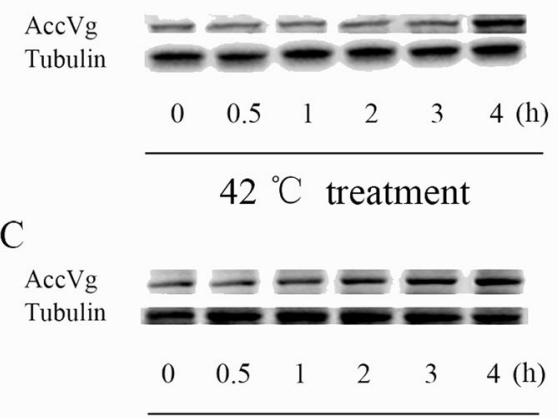

Phoxim treatment
B

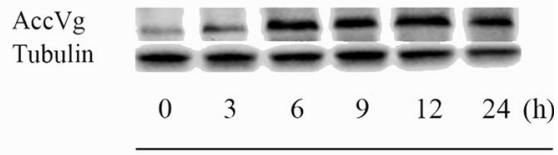

$\mathrm{CdCl}_{2}$ treatment

$\mathrm{D}$

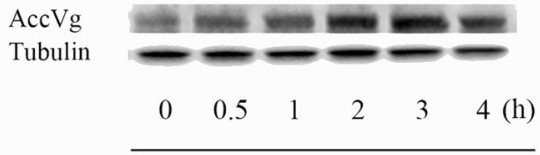

Paraquat treatment

Figure 7 Western blot analysis of $V g$ in different treatments of A. cerana cerana workers. SDS-PAGE (7.5\%) was performed with $80 \mathrm{ng}$ of total proteins from workers. Images are representative of three replicates.

increased and reached the highest level at $9 \mathrm{~h}$; extension of the treatment time resulted in decreased expression. Many heavy metals, including $\mathrm{CdCl}_{2}$ (Liu et al., 2009), can induce the formation of endogenous ROS, which include superoxide anions, hydrogen peroxide and hydroxyl radicals. Endogenously generated ROS should be kept in balance, as high ROS concentration can damage DNA, protein and lipids (Narendra et al., 2007). In this study, $\mathrm{Acc} V \mathrm{~g}$ expression increased rapidly, peaking at $9 \mathrm{~h}$, and then decreased in response to heavy metal exposure. The increased AccVg expression following $\mathrm{CdCl}_{2}$ exposure could enhance the tolerance of $A$. cerana cerana to oxidative stress. Considering our result, we hypothesised that heavy metals (such as $\mathrm{CdCl} 2$ ) could cause stress and induce the expression of $\mathrm{AccVg}$ through distinct pathways.

A large number of putative HSF binding sites were identified in the $\mathrm{AccVg}$ 5'-flanking region that are likely to participate in heat-induced transcriptional activation. Western blot analysis of AccVg after the $42{ }^{\circ} \mathrm{C}$ treatment confirmed the result, suggesting that $\mathrm{AccVg}$ might play a role in response to extreme temperature. Pesticides were the main threat to a honeybee's life. Pesticide treatments, which induce the formation of reactive oxygen species, resulted in an increase in the expression of AccVg (Fig. 6) (Day et al., 1999). Bus et al. (1974) noted that paraquat induced oxidative stress by redox cycling with various cellular diaphorases and oxygen to produce superoxide radicals. Our data suggest that Vg activity protects the bee against oxidative damage induced by pesticides. AccVg is preferentially oxidized, which is a property that is indicative of an antioxidant function (Quinlan et al., 2005; Salo et al., 1988; Andrus et al., 1998). With regard to protein expression, the western blot results also indicated the response of AccVg to various oxidative stresses (Fig. 7). All the results demonstrated that $\mathrm{AccVg}$ would be induced and activated after abiotic stress, which indicated that AccVg quite possibly plays a significant role in the response of $A$. cerana cerana to adverse environmental stresses. Though some of these stressors also cause increases of gene expression in A. cerana cerana, the transcript levels and patterns are quite different from those of the AccVg protein levels.

In conclusion, we identified that the $V g$ gene in A. cerana cerana cloned the 5 '-flanking region and predicted putative binding sites for transcription factors. Next, the patterns of $V g$ expression during different developmental stages and under different adverse stresses were examined at the transcriptional level. Then, the expression patterns at the protein level were examined to confirm these results. All of these results raise the possibility that AccVg plays a role in the response to adverse stress. To further understand the characteristics of $\mathrm{AccVg}$, additional studies that directly address the functional significance of this gene and its products should be carried out. 


\section{ACKNOWLEDGMENTS}

This work was financially supported by the China Agriculture Research System (No. CARS-45), the National Natural Science Foundation of China (No. 31572470) and Shandong Province Agricultural Fine Varieties Breeding Projects (2014-2016).

\section{Author's Contributions}

Baohua Xu and Xingqi Guo designed the experiments; Hongfang Wang and Ying Wang participated in the design and Lanting Ma, Zhenguo Liu and Ming Zhu performed experiments.

Clonage moléculaire, expression et réponse au stress oxydatif du gène de la vitellogénine $(\mathrm{AccVg})$ d'Apis cerana cerana

Apidae / stade de développement / stress abiotique / PCR quantitative en temps réel / interférence ARN

Klonierung, Expression und oxidative Stressantwort des Apis cerana cerana Vitellogenin-Gens (AccVg)

Vitellogenin / Apis cerana cerana / RNA-Interferenz / oxidative Stressantwort / RT-PCR

\section{REFERENCES}

Amdam, G.V., Omholt, S.W. (2002) The regulatory anatomy of honeybee lifespan. J. Theor. Biol. 216, 209-228.

Amdam, G.V., Simões, Z.L., Hagen, A., Norberg, K., Schrøder, K., Mikkelsen, Ø., Kirkwood, T.B., Omholt, S.W. (2004) Hormonal control of the yolk precursor vitellogenin regulates immune function and longevity in honeybees. Exp. Gerontol. 39, 767-773.

Andrus, P.K., Fleck, T.J., Gurney, M.E., Hall, E.D. (1998) Protein oxidative damage in a transgenic mouse model of familial amyotrophic lateral sclerosis. J. Neurochem. 71, 2041-2048.

Balaban, R.S., Nemoto, S., Finkel, T. (2005) Mitochondria, oxidants, and aging. Cell 120, 483-495.

Barchuk, A. R., Bitondi, M. M., Simões, Z. L. (2002) Effects of juvenile hormone and ecdysone on the timing of vitellogenin appearance in hemolymph of queen and worker pupae of Apis mellifera. J. Insect Sci. 2 (1), 1.

Bitondi, M., Simoes, Z.P. (1996) The relationship between level of pollen in the diet, vitellogenin and juvenile hormone titres in Africanized Apis mellifera workers. J. Apic. Res. 35, 27-36.
Bus, J.S., Aust, S.D., Gibson, J.E. (1974) Superoxide- and singlet oxygen-catalyzed lipid peroxidation as a possible mechanism for paraquat (methyl viologen) toxicity. Biochem. Biophys. Res. Commun. 58, 749-755.

Corona, M., Velarde, R.A., Remolina, S., Moran-Lauter, A., Wang, Y., Hughes, K.A., Robinson, G.E. (2007) Vitellogenin, juvenile hormone, insulin signaling, and queen honey bee longevity. Proc. Natl. Acad. Sci. USA 104, 7128-7133.

Day, B.J., Patel, M., Calavetta, L., Chang, L.-Y., Stamler, J.S. (1999) A mechanism of paraquat toxicity involving nitric oxide synthase. Proc. Natl. Acad. Sci USA 96, 12760-12765.

Ding, Y., Hawkes, N., Meredith, J., Eggleston, P., Hemingway, J., Ranson, H. (2005) Characterization of the promoters of epsilon glutathione transferases in the mosquito Anopheles gambiae and their response to oxidative stress. Biochem. J. 387, 879-888.

Dröge, W. (2002) Free radicals in the physiological control of cell function. Physiol. Rev. 82, 47-95.

Engels, W., Kaatz, H., Zillikens, A. (1990) Honey bee reproduction: vitellogenin and caste-specific regulation of fertility. Invertebr. Reprod. D 5, 495-502.

Fleig, R. (1995) Role of the follicle cells for yolk uptake in ovarian follicles of the honey bee Apis mellifera L. (hymenoptera: Apidae). Int. J. Insect Morphol. 24 (95), 427-433.

Goto, S., Nakamura, A., Radak, Z., Nakamoto, H., Takahashi, R., Yasuda, K., Sakurai, Y., Ishii, N. (1999) Carbonylated proteins in aging and exercise: immunoblot approaches. Mech. Ageing Dev. 107, 245-253.

Guidugli, K. R., Piulachs, M., Bellés, X. (2005) Vitellogenin expression in queen ovaries and in larvae of both sexes of Apis mellifera. Archiv. Insect Biochem. 59 (4), 211-218.

Hartfelder, Engels, W. (1998) Social insect polymorphism: hormonal regulation of plasticity in development and reproduction in the honeybee. Curr. Top. Dev. Biol. 40, 45-77

Hayward, A., Takahashi, T., Bendena, W.G., Tobe, S.S., Hui, J.H. (2010) Comparative genomic and phylogenetic analysis of vitellogenin and other large lipid transfer proteins in metazoans. Febs. Lett. 584, 1273-1278.

Kottuparambil, S., Shin, W., Brown, M.T., Han, T. (2012) UV-B affects photosynthesis, ROS production and motility of the freshwater flagellate, Euglena agilis Carter. Aquat. Toxicol. 122, 206-213.

Livak, K. J., Schmittgen T. D. (2001) Analysis of relative gene expression data using real-time quantitative PCR and the 2(-Delta Delta C (T)) method. J. Virol. Methods 25 (4): 402-408.

Liu, J., Qu, W., Kadiiska, M.B. (2009) Role of oxidative stress in cadmium toxicity and carcinogenesis. Toxicol. Appl. Pharmacol. 238, 209-214.

Lushchak, V.I. (2011) Environmentally induced oxidative stress in aquatic animals. Aquat. Toxicol. 101, 13-30.

Michelette, E. R. D. F., Soares, A. E. E. (1993) Characterization of preimaginal developmental stages in 
Africanized honey bee workers (Apis mellifera L). Apidologie 24 (4):431-440.

Narendra, M., Bhatracharyulu, N., Padmavathi, P., Varadacharyulu, N. (2007) Prallethrin induced biochemical changes in erythrocyte membrane and red cell osmotic haemolysis in human volunteers. Chemosphere 67, 1065-1071.

Pham-Delègue, M. H., Girard, C., Métayer, M. L. (2000) Long-term effects of soybean protease inhibitors on digestive enzymes, survival and learning abilities of honeybees. Entomol. Exp. Appl. 95 (1), 21-29.

Piulachs, M. D., Guidugli, K. R., Barchuk, A. R. (2003) The vitellogenin of the honey bee, Apis mellifera: structural analysis of the cDNA and expression studies. Insect Biochem. Mol. Biol. 33 (4), 459-65.

Quinlan, G.J., Martin, G.S., Evans, T.W. (2005) Albumin: biochemical properties and therapeutic potential. Hepatology 41, 1211-1219.

Salo, D. C., Lin, S. W., Pacifici, R. E. (1988) Superoxide dismutase is preferentially degraded by a proteolytic system from red blood cells following oxidative modification by hydrogen peroxide. Free Radic. Biol. Med. 5 (s 5-6), 335-339.

Seehuus, S.C., Norberg, K., Gimsa, U., Krekling, T., Amdam, G.V. (2006) Reproductive protein protects functionally sterile honey bee workers from oxidative stress. Proc. Natl. Acad. Sci USA 103, 962-967.

Spieth, J., Nettleton, M., Zucker-Aprison, E., Lea, K., Blumenthal, T. (1991) Vitellogenin motifs conserved in nematodes and vertebrates. J. Exp. Zool. Part. B 32, 429-438.
Tang-Feldman, Y. J., Wojtowicz, A., Lochhead, G. R. (2006) Use of quantitative real-time PCR (qRT-PCR) to measure cytokine transcription and viral load in murine cytomegalovirus infection. J. Virol. Methods 131 (2), 122-129.

Trenczek, T., Zillikens, A., Engels, W. (1989) Developmental patterns of vitellogenin haemolymph titre and rate of synthesis in adult drone honey bees (Apis mellifera). J. Insect Physiol. 35, 475-481.

Tseng, D.-Y., Chen, Y.-N., Kou, G.-H., Lo, C.-F., Kuo, C.M. (2001) Hepatopancreas is the extraovarian site of vitellogenin synthesis in black tiger shrimp, Penaeus monodon. Comp. Biochem. Physiol. A Mol. Integr. Physiol. 129, 909-917.

Tufail, M., Takeda, M. (2008) Molecular characteristics of insect vitellogenins. J. Insect Physiol. 54, 1447-1458.

Wheeler, D.E., Kawooya, J.K. (1990) Purification and characterization of honey bee vitellogenin. Archiv. Insect Biochem. 14, 253-267.

Winston, M. (1987) The Biology of the Honey Bee. Harvard University Press 35 (35), 316-318.

Yang, L.-H., Huang, H., Wang, J.-J. (2010) Antioxidant responses of citrus red mite, Panonychus citri (McGregor) (Acari: Tetranychidae), exposed to thermal stress. J. Insect Physiol. 56, 1871-1876.

Zhang, Y., Yan, H., Lu, W., Li, Y., Guo, X., Xu, B. (2013) A novel Omega-class glutathione S-transferase gene in Apis cerana cerana: molecular characterisation of GSTO2 and its protective effects in oxidative stress. Cell. Stress Chaperon 18, 503-516. 